\title{
Limits of dihedral groups
}

\author{
Luc Guyot
}

Received: 22 May 2008 / Accepted: 25 November 2009 / Published online: 11 December 2009

(C) The Author(s) 2009. This article is published with open access at Springerlink.com

\begin{abstract}
We characterise limits of dihedral groups in the space of finitely generated marked groups. We also describe the topological closure of dihedral groups in the space of marked groups on a fixed number of generators.
\end{abstract}

Keywords Space of marked groups · Dihedral groups · Universal theory · Ultrafilters

Mathematics Subject Classification (2000) $\quad$ 20F05 · 54G12 · 11U09

\section{Introduction}

The space of marked groups is a topological object which enables us to talk about approximation between groups and algebraic limit processes in terms of convergence. It can be defined as the set of normal subgroups of a given free group $\mathbb{F}$ with the topology induced by the product topology of $\{0,1\}^{\mathbb{F}}$. The idea of topologizing the set of subgroups of a given group goes back to Chabauty's topology on the closed subgroups of a locally compact group [3]. However, it was Gromov who first gave some properties of a topology on a set of finitely generated groups to prove growth results [9]. The commonly used definition of the space of marked groups on $m$ generators was given by Grigorchuk [7]. This compact, totally disconnected and metrisable space has been used to prove the existence of infinite groups with unexpected or rare properties $[4,17,18]$.

This work was supported by the Swiss National Science Foundation Grant $\sharp$ PP002-68627.

L. Guyot $(\bowtie)$

Georg-August University, Bunsenstrasse 3-5, 37073 Goettingen, Germany

e-mail: guyot@uni-math.gwdg.de 
The space of marked groups is the topological framework used by Champetier and Guirardel [2] in their new approach to Sela's limit groups ${ }^{1}$ [16]. They interpret limit groups as limits of free groups with respect to Chabauty's topology. By linking topology and logic, they give the first proof of the fact that limit groups are fully residually free groups without using finite presentability (see [10-12,16] for a proof of finite presentability of limit groups). Their methods give a simple proof that a finitely generated group is a limit group if and only if it has the same universal theory as a free group of rank two. In the present paper, we use the links between convergence and logic to study limits of dihedral groups. Our motivation is the following:

Problem [2,6] Describe the topological closure of the set of finite groups in the space of marked groups.

We carry out such a description for the most elementary finite groups: cyclic and dihedral finite groups.

The dihedral group $\mathbb{D}_{2 n}:=\left\langle a, b \mid a^{2}=b^{n}=1, a^{-1} b a=b^{-1}\right\rangle$ is a group of order $2 n$ for every $n \geq 1$. If we omit the relation $b^{n}=1$, we obtain a presentation of $\mathbb{D}_{\infty}$, the infinite dihedral group. The groups $\mathbb{D}_{2}$ (which is isomorphic to $\mathbb{Z} / 2 \mathbb{Z}$ ) and $\mathbb{D}_{4}$ (which is isomorphic to the Vierergruppe $\mathbb{Z} / 2 \mathbb{Z} \times \mathbb{Z} / 2 \mathbb{Z}$ ) are the only abelian dihedral groups. The subgroup $\langle b\rangle$ generated by $b$ is a cyclic normal subgroup of order $n$ (possibly infinite) on which $\langle a\rangle$ acts by conjugation. Thus $\mathbb{D}_{2 n}$ is isomorphic to the semi-direct product $\mathbb{Z} / n \mathbb{Z} \rtimes \mathbb{Z} / 2 \mathbb{Z}$ where the action of $\mathbb{Z} / 2 \mathbb{Z}$ on $\mathbb{Z} / n \mathbb{Z}$ is the multiplication by -1 . Observe that proper quotients of $\mathbb{D}_{\infty}$ are finite dihedral groups and that any such group is a quotient of $\mathbb{D}_{\infty}$.

We denote by $\mathscr{M}$ the space of all finitely generated marked groups (see Sect. 2 for a definition). Unless otherwise stated, limits of groups are limits with respect to Chabauty's topology on $\mathscr{M}$.

Let $P$ be a group-theoretic property. A group $G$ is fully residually $P$ if for any finite subset $F$ of $G \backslash\{1\}$ there is a group $H$ satisfying $P$ and a homomorphism from $G$ to $H$ that maps no element of $F$ to the trivial element. We refer the reader to Sect. 2 for the definitions appearing in our first theorem (convergence in $\mathscr{M}$, universal theory $T h_{\forall}(G)$ of $G$, ultrafilter and ultraproduct). The only abelian limits of dihedral groups are easily seen to be the marked groups abstractly isomorphic to $\mathbb{D}_{2}$ or $\mathbb{D}_{4}$ (see Sect. 2). In Sect. 4 , we characterise non-abelian limits in the following way:

Theorem A (Theorem 4.1) Let $G$ be a non-abelian finitely generated group. The following conditions are equivalent:

(lim) $G$ is a limit of dihedral groups;

(res) $G$ is fully residually dihedral;

(iso) $G$ is isomorphic to a semi-direct product $A \rtimes \mathbb{Z} / 2 \mathbb{Z}$ where $A$ is a limit of cyclic groups $^{2}$ on which $\mathbb{Z} / 2 \mathbb{Z}$ acts by multiplication by -1 ;

$\left(T h_{\forall}\right) T h_{\forall}(G) \supset \bigcap_{n \geq 3} T h_{\forall}\left(\mathbb{D}_{2 n}\right)$;

$(\Pi / \mathfrak{U}) \quad G$ isomorphically embeds into $\left(\prod_{n \geq 3} \mathbb{D}_{2 n}\right) / \mathfrak{U}$ for some ultrafilter $\mathfrak{U}$ on $\mathbb{N}$.

We denote by $\omega$ the smallest infinite ordinal, i.e., the set $\mathbb{N}$ of positive integers endowed with its natural order. In Sect. 5 we describe the set of limits of dihedral groups on $m$ generators:

1 The class of limit groups coincides with the long-studied class of fully residually free groups. These groups play a key role in the theory of equations over free groups.

2 Proposition 4.2 shows that limits of cyclic groups are finitely generated abelian groups with cyclic torsion subgroup. 
Theorem B (Theorem 5.2) The topological closure $\mathscr{D}_{m}$ of dihedral marked groups in $\mathscr{M}_{m}$ is homeomorphic to $\omega^{m-1}\left(2^{m}-1\right)+1$ endowed with the order topology.

In other words, $\mathscr{D}_{m}$ is the disjoint union of $2^{m}-1$ copies of $\overline{\mathbb{N}}^{m-1}$ where $\overline{\mathbb{N}}=\mathbb{N} \cup\{\infty\}$ is the Alexandroff compactification of $\mathbb{N}$. We use a theorem of Mazurkiewicz and Sierpinski [13] on Cantor-Bendixson invariants of countable compact spaces to prove the last result. For comparison, the set of abelian marked groups on $m$ generators is homeomorphic to $\omega^{m}+1$. This fact can be easily derived from the proof of Theorem B.

The first three sections deal with background material. Sections 4 and 5 present the proofs of Theorems A and B, respectively. Section 6 is a discussion on the space of countable marked groups.

\section{Convergence and logic}

In this section, we will give preliminary definitions and results needed in the proofs of the main theorems. We first define marked groups and the topology (called Chabauty's topology, Cayley's topology or the weak topology) on the set of marked groups. Second, we relate convergence in the space of marked groups to universal theory and ultraproducts (see [2] for a more detailed presentation).

Definition 2.1 The pair $(G, S)$ is a marked group on $m$ generators if $G$ is a group and $S=\left(g_{1}, \ldots, g_{m}\right)$ is an ordered system of generators of $G$. We call also $S$ a generating $G$-vector of length $m$ (or simply a marking of $G$ of length $m$ ) and we denote by $V(G, m)$ the set of all these generating $G$-vectors.

We denote by $r(G)$ the rank of $G$, i.e., the smallest number of generators of $G$. Two marked groups $(G, S)$ and $\left(G^{\prime}, S^{\prime}\right)$ (with $S=\left(g_{1}, \ldots, g_{m}\right)$ and $S^{\prime}=\left(g_{1}^{\prime}, \ldots, g_{m}^{\prime}\right)$ ) are equivalent if there is an isomorphism $\phi: G \longrightarrow G^{\prime}$ such that $\phi\left(g_{i}\right)=g_{i}^{\prime}$ for $i=1, \ldots, m$.

Nota Bene 2.2 We denote also by $(G, S)$ the equivalence class of $(G, S)$ and we call this class a marked group.

Let $\mathbb{F}_{m}$ be the free group with basis $\left(e_{1}, \ldots, e_{m}\right)$ and let $\mathbb{F}_{\infty}$ the free group with basis $\left(e_{i}\right)_{i \geq 1}$. Let $G$ and $G^{\prime}$ be two groups and let $p: \mathbb{F}_{m} \rightarrow G$ and $p^{\prime}: \mathbb{F}_{m} \rightarrow G^{\prime}$ be two epimorphisms. The epimorphisms $p$ and $p^{\prime}$ are equivalent if there is an isomorphism $\phi$ such that the following diagram commutes:

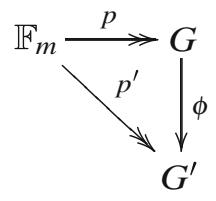

Using the universal property of $\mathbb{F}_{m}$, we establish a natural bijection from marked groups on $m$ generators to equivalence classes of epimorphisms with source $\mathbb{F}_{m}$. The last set is clearly in one-one correspondence with the set $\mathscr{N}\left(\mathbb{F}_{m}\right)$ of all normal subgroups of $\mathbb{F}_{m}$. We endow $\mathscr{N}\left(\mathbb{F}_{m}\right)$ with the topology induced by the Tychonoff topology on the product $\{0,1\}^{\mathbb{F}_{m}}$ which identifies with the set of all subsets of $\mathbb{F}_{m}$. The following lemma shows that $\mathscr{N}\left(\mathbb{F}_{m}\right)$ is a closed subspace of $\{0,1\}^{\mathbb{F}_{m}}$. 
Lemma 2.3 Let $\left(A_{i}\right)_{i}$ be a sequence of subsets of $\mathbb{F}_{m}$. We set $\lim \inf A_{i}=\bigcup_{i} \bigcap_{i \geq j} A_{i}$ and $\lim \sup A_{i}=\bigcap_{i} \bigcup_{i \geq j} A_{i}$. The sequence $\left(A_{i}\right)_{i}$ converges to $A$ in $\{0,1\}^{\mathbb{F}_{m}}$ if and only if $A=\lim \inf A_{i}=\lim \sup A_{i}$.

Thus the set $\mathscr{M}_{m}$ of marked groups on $m$ generators, which identifies with $\mathbb{N}\left(\mathbb{F}_{m}\right)$, is endowed with a compact, metrisable and totally discontinuous topology. We have for instance $\left(\mathbb{Z} / k \mathbb{Z},[1]_{k}\right) \underset{k \rightarrow \infty}{\longrightarrow}(\mathbb{Z}, 1)$ in $\mathscr{M}_{1}$ and $\left(\mathbb{D}_{2 k},(a, b)\right) \underset{k \rightarrow \infty}{\longrightarrow}\left(\mathbb{D}_{\infty},(a, b)\right)$ in $\mathscr{M}_{2}$ with respect to Chabauty's topology.

Let $G$ be a finitely generated group. We define $\mathscr{N}(G)$ as the set of normal subgroups of $G$ endowed with the topology induced by $\{0,1\}^{G}$. Let $p: \mathbb{F}_{m} \rightarrow G$ be an epimorphism and let $p^{*}: \mathscr{N}(G) \longrightarrow \mathscr{N}\left(\mathbb{F}_{m}\right)$ be defined by $p^{*}(N)=p^{-1}(N)$. The map $p^{*}$ is clearly continuous and injective.

Lemma 2.4 [2, Lemma 2.2] If $G$ is finitely presented then $p^{*}$ is an open embedding.

Thus, for any marking $S$ of a finitely presented group $G$ in $\mathscr{M}_{m}$, there is a neighborhood of $(G, S)$ containing only quotients of $G$ (the quotient map is induced by the natural bijection between the markings). Namely, this is the set corresponding to the image of $p^{*}$ where $p$ is the epimorphism defined by means of $S$.

Remark 2.5 It follows from Lemma 2.4 that finite groups are isolated in $\mathscr{M}$. Isolated groups are characterised in $[5,8]$.

From the spaces $\mathscr{M}_{m}$, we build up the space $\mathscr{M}$ of all finitely generated marked groups. Observe first that the map $\left(G,\left(g_{1}, \ldots, g_{m}\right)\right) \mapsto\left(G,\left(g_{1}, \ldots, g_{m}, 1\right)\right)$ defines a continuous and open embedding $i_{m}: \mathscr{M}_{m} \hookrightarrow \mathscr{M}_{m+1}$. The inductive limit $\mathscr{M}$ of the system $\left\{i_{m}: \mathscr{M}_{m} \hookrightarrow\right.$ $\left.\mathscr{M}_{m+1}\right\}_{m \geq 1}$ is a metrisable locally compact and totally discontinuous space. Observe that convergence in $\mathscr{M}$ boils down to convergence in $\mathscr{M}_{m}$ for some $m$. The space $\mathscr{M}$ can be viewed as the set of (equivalence classes) of groups marked with an infinite sequence of generators which are eventually trivial. Let $v_{1}, \ldots, v_{k}, w_{1}, \ldots, w_{l}$ be elements of $\mathbb{F}_{\infty}$ and let $(\Sigma)$ be the system

$$
\left\{\begin{array}{l}
v_{1}=1, \ldots, v_{k}=1 \\
w_{1} \neq 1, \ldots, w_{l} \neq 1
\end{array}\right.
$$

We denote by $O_{\Sigma}$ the set of marked groups $(G, S)$ of $\mathscr{M}$ for which $S$ (possibly completed by trivial elements) is a solution of $(\Sigma)$ in $G$. The family of sets $O_{\Sigma}$ defines a countable basis of open and closed subsets of $\mathscr{M}$.

Remark 2.6 Let $P$ be group theoretic property stable under taking subgroups and let $G$ be a fully residually $P$ group. Using the previous basis, we easily deduce that for any marking $S$ of $G,(G, S)$ is the limit in $\mathscr{M}$ of a sequence of marked groups with $P$. The converse is also true when $G$ is finitely presented by Lemma 2.4 .

Lemma 2.7 (Subgroup and convergence [2, Proposition 2.20]) Let $\left(G_{n}, S_{n}\right)_{n}$ be a convergent sequence in $\mathscr{M}$ with limit $(G, S)$. Let $H$ be a finitely generated subgroup of $G$. Then, for any marking $T$ of $H$ there is a sequence of marked groups $\left(H_{n}, T_{n}\right)_{n}$ which converges in $\mathscr{M}$ to $(H, T)$ and such that $H_{n}$ is a subgroup of $G_{n}$.

It is important to note that being a limit in $\mathscr{M}$ of marked groups with a given property $P$ does not depend on the marking: 
Lemma 2.8 [5, Lemma 1] Let $(G, S)$ and $(H, T)$ be in $\mathscr{M}$. Assume that $G$ and $H$ are abstractly isomorphic. Then we can find a neighborhood $U$ of $(G, S)$, a neighborhood $V$ of $(H, T)$, and a homeomorphism $\phi: U \longrightarrow V$ mapping $(G, S)$ onto $(H, T)$. Moreover, $\phi$ preserves the isomorphism relation.

Thus, being a limit of finite (or equally, free, cyclic, dihedral) groups is a group property that does not depend on the marking. As $\mathbb{D}_{\infty}$ is a limit of finite dihedral groups, limits of dihedral groups are all limits of finite ones.

Let $G$ be a finite group. For all $m \geq r(G)$, there are only finitely many marked groups isomorphic to $G$ in $\mathscr{M}_{m}$ (there are only finitely many equivalence classes of epimorphisms $\left.p: \mathbb{F}_{m} \rightarrow G\right)$. Hence any compact subset of $\mathscr{M}$ contains only finitely many marked groups isomorphic to $G$. Since the property of being abelian is open in $\mathscr{M}$ by Lemma 2.4, abelian limits of dihedral groups are limits of abelian dihedral groups $\mathbb{D}_{2}$ or $\mathbb{D}_{4}$. These limits are consequently isomorphic either to $\mathbb{D}_{2}$ or $\mathbb{D}_{4}$. For this reason, we only consider non-abelian limits.

We now turn to relations between convergence and logic. We fix a countable set of variables $\left\{x_{1}, x_{2}, \ldots\right\}$ and the set of symbols $\{\wedge, \vee, \neg, \forall,(),, \cdot,-1,=, 1\}$ that stand for the usual logic and group operations. We consider the set $T h_{\forall}$ of universal sentences in group theory written using the above variables, i.e., all formulas of the type $\forall x_{1} \ldots \forall x_{k} \phi\left(x_{1}, \ldots, x_{k}\right)$, where $\phi\left(x_{1}, \ldots, x_{k}\right)$ is a quantifier free formula built up from variables and logic symbols (see [1,2] for a precise definition). For instance, $\forall x \forall y(x y=y x)$ is a universal sentence that is true in any abelian group while $\forall x\left(x=1 \vee x^{2} \neq 1\right)$ expresses that there is no 2-torsion in a group. If a universal sentence $\sigma$ is true in $G$ (we also say that $G$ satisfies $\sigma$ ), we write $G \models \sigma$. We denote by $T h_{\forall}(G)$ the set of universal sentences which are true in $G$. Let $\left(A_{n}\right)_{n}$ be a sequence of subsets of $T h_{\forall}$. We set $\lim \sup A_{n}:=\bigcap_{n} \bigcup_{k \geq n} A_{k}$ and $\lim \inf A_{n}:=\bigcup_{n} \bigcap_{k \geq n} A_{k}$. The set of marked groups of $\mathscr{M}$ satisfying a given family of universal sentences defines a closed subset. More precisely, quoting Proposition 5.2 of [2] and reformulating slightly Proposition 5.3, we have:

Proposition 2.9 (Limits and universal theory) Let $G$ be a finitely generated group and let $\left(G_{n}\right)_{n}$ be a sequence of finitely generated groups.

(i) Assume that $(G, S)$ is the limit in $\mathscr{M}$ of $\left(G_{n}, S_{n}\right)_{n}$ for some marking $S$ of $G$ and some ordered generating set $S_{n}$ of $G_{n}$. Then $T h_{\forall}(G) \supset \lim \sup T h_{\forall}\left(G_{n}\right)$.

(ii) Assume $T h_{\forall}(G) \supset \bigcap_{n} T h_{\forall}\left(G_{n}\right)$. Then, for any ordered generating set $S$ of $G$, there is some integer sequence $\left(n_{k}\right)_{k}$ such that $(G, S)$ is the limit in $\mathscr{M}$ of some sequence $\left(H_{k}, T_{k}\right)$ satisfying $H_{k} \leq G_{n_{k}}$.

It directly follows that a variety of groups (see [14] for a definition) defines closed subspaces of $\mathscr{M}_{m}$ and $\mathscr{M}$. For example, limits of dihedral groups are metabelian groups because dihedral groups are metabelian.

Convergence in $\mathscr{M}$ can also be related to ultraproducts.

Definition 2.10 (Ultrafilter) An ultrafilter $\mathfrak{U}$ on $\mathbb{N}$ is a finitely additive measure with total mass 1 which takes values in $\{0,1\}$. In other words, it is a map from $\mathcal{P}(\mathbb{N})$ (the set of all subsets of $\mathbb{N}$ ) to $\{0,1\}$ satisfying $\mathfrak{U}(\mathbb{N})=1$ and such that, for all disjoint subsets $A$ and $B$ of $\mathbb{N}$, we have $\mathfrak{U}(A \cup B)=\mathfrak{U}(A)+\mathfrak{U}(B)$.

Let $\mathfrak{U}$ be an ultrafiler on $\mathbb{N}$ and let $\left(G_{n}\right)_{n}$ be sequence of groups. There is a natural relation on the cartesian product $\prod_{n} G_{n}:\left(g_{n}\right)_{n}$ and $\left(h_{n}\right)_{n}$ are equal $\mathfrak{U}$-almost everywhere if $\mathfrak{U}\left(\left\{n \in \mathbb{N} \mid g_{n}=g_{n}^{\prime}\right\}\right)=1$. 
Definition 2.11 (Ultraproduct) The ultraproduct of the sequence $\left(G_{n}\right)_{n}$ relative to $\mathfrak{U}$ is the quotient of $\prod_{n} G_{n}$ by the equivalence relation of equality $\mathfrak{U}$-almost everywhere. We denote it by $\left(\prod_{n} G_{n}\right) / \mathfrak{U}$.

Let $\left(A_{n}\right)_{n}$ be a sequence of subsets of $T h_{\forall}$. We set

$$
\lim _{\mathfrak{U}} A_{n}:=\left\{\phi \in T h_{\forall} \mid \phi \text { belongs to } A_{n} \text { for } \mathfrak{U}-\text { almost every } n\right\} .
$$

We have:

Theorem 2.12 (Lǒs [1]) $T h_{\forall}\left(\left(\prod_{n} G_{n}\right) / \mathfrak{U}\right)=\lim _{\mathfrak{U}} T h_{\forall}\left(G_{n}\right)$.

An ultrafilter is said to be principal if it is a Dirac mass. The following proposition relates convergence in $\mathscr{M}$ to ultraproducts:

Proposition 2.13 (Limits and ultraproducts [2, Prop. 6.4])

Let $(G, S)$ be in $\mathscr{M}$ and let $\left(G_{n}, S_{n}\right)_{n}$ be a sequence in $\mathscr{M}$.

(i) If $\left(G_{n}, S_{n}\right)_{n}$ accumulates on $(G, S)$ in $\mathscr{M}$, then $G$ isomorphically embeds into $\left(\prod_{n} G_{n}\right) / \mathfrak{U}$ for some non-principal ultrafilter $\mathfrak{U}$.

(ii) If $\left(G_{n}, S_{n}\right)_{n}$ converges to $(G, S)$ in $\mathscr{M}$, then $G$ isomorphically embeds into $\left(\prod_{n} G_{n}\right) / \mathfrak{U}$ for all non-principal ultrafilters $\mathfrak{U}$.

(iii) Let $H$ be a finitely generated group. If $H$ isomorphically embeds into $\left(\prod_{n} G_{n}\right) / \mathfrak{U}$ for some non-principal ultrafilter $\mathfrak{U}$, then for all markings $T$ of $H$, we can find a sequence of integers $\left(n_{k}\right)_{k}$ and a sequence $\left(H_{k}, T_{k}\right)_{k}$ that converges to $(H, T)$ in $\mathscr{M}$ and such that $H_{k} \leq G_{n_{k}}$ for all $k$.

\section{Cantor-Bendixson invariants}

This section deals with the basics of Cantor-Bendixson analysis needed in the proof of Theorem 5.2. Let $X$ be a topological space. We denote by $X^{\prime}$ the set of accumulation points of $X$. Let $X^{(0)}:=X$. We define by transfinite induction the $\alpha$-th derived set of $X: X^{(\alpha)}=\left(X^{(\alpha-1)}\right)^{\prime}$ if $\alpha$ is a successor and $X^{(\alpha)}=\bigcap_{\beta<\alpha} X^{(\beta)}$ if $\alpha$ is a limit ordinal. We denote by $\omega$ the set $\mathbb{N}$ of integers endowed with its natural order. We use the following topological classification theorem:

Theorem 3.1 (Mazurkiewicz-Sierpinski Theorem [13]) For any given pair $(\alpha, n)$ where $\alpha$ is a countable ordinal number and $n$ belongs to $\mathbb{N}$, there is (up to homeomorphism) a unique countable compact space $X$ such that $X^{(\alpha)}$ has exactly $n$ points: the set $\omega^{\alpha} n+1$ endowed with the order topology.

The pair $(\alpha, n)$ is the characteristic system of $X$. For example, the Alexandroff compactification $\overline{\mathbb{N}}$ of $\mathbb{N}$ is homeomorphic to $\omega+1$. Its characteristic system is then $(1,1)$. Similarly, the characteristic system of $\overline{\mathbb{N}}^{k}$ is $(k, 1)$. The Cantor-Bendixson rank of a point $x$ in $X$ is the smallest ordinal number $\alpha$ such that $x$ does not belong to $X^{(\alpha)}$. A countable compact space has the characteristic system $(\alpha, n)$ if and only if the set of points of maximal Cantor-Bendixson rank (i.e., rank $\alpha$ ) has cardinality $n$. Observe that the Cantor-Bendixson rank of $(G, S)$ in $\mathscr{M}_{m}$ is the Cantor-Bendixson rank of $(G, S)$ in $\mathscr{M}$ and does not depend on $S$ because of Lemma 2.8 . 


\section{Characterisation of limits}

Theorem 4.1 Let G be a non-abelian finitely generated group. The following conditions are equivalent:

(lim) $G$ is a limit of dihedral groups;

(res) $G$ is fully residually dihedral;

(iso) $G$ is isomorphic to a semi-direct product $A \rtimes \mathbb{Z} / 2 \mathbb{Z}$ where $A$ is a limit of cyclic groups on which $\mathbb{Z} / 2 \mathbb{Z}$ acts by multiplication by -1 ;

$\left(T h_{\forall}\right) T h_{\forall}(G) \supset \bigcap_{n \geq 3} T h_{\forall}\left(\mathbb{D}_{2 n}\right)$;

$(\Pi / \mathfrak{U}) \quad G$ isomorphically embeds into $\left(\prod_{n \geq 3} \mathbb{D}_{2 n}\right) / \mathfrak{U}$ for some ultrafilter $\mathfrak{U}$ on $\mathbb{N}$.

We first characterise limits of cyclic groups. The following characterisation will be used in the proof of Theorem 4.1 .

Proposition 4.2 Let $G$ be a finitely generated group. The following conditions are equivalent:

$(\lim )_{c} G$ is a limit of cyclic groups;

$(\mathrm{res})_{c} G$ is fully residually cyclic;

$(\text { iso })_{c} G$ is isomorphic to an abelian group with cyclic torsion subgroup;

$\left(T h_{\forall}\right)_{c} T h_{\forall}(G) \supset \bigcap_{n \geq 1} T h_{\forall}(\mathbb{Z} / n \mathbb{Z})$;

$(\Pi / \mathfrak{U})_{c} G$ isomorphically embeds into $\left(\prod_{n \geq 1} \mathbb{Z} / n \mathbb{Z}\right) / \mathfrak{U}$ for some ultrafilter $\mathfrak{U}$ on $\mathbb{N}$.

We now show Proposition 4.2 and then Theorem 4.1.

\section{Proof of Proposition 4.2.}

Here is the logical scheme of the proof:

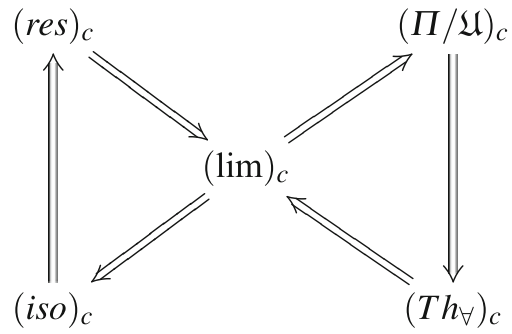

We begin with the right triangle of implications.

$(\lim )_{c} \Longrightarrow(\Pi / \mathfrak{U})_{c}$ : we first assume that there is a sequence $\left(\mathbb{Z} / n \mathbb{Z}, S_{n}\right)_{n}$ in $\mathscr{M}$ which accumulates on $(G, S)$ for some marking $S$ of $G$. Then $G$ embeds isomorphically into $\left(\prod_{n>1} \mathbb{Z} / n \mathbb{Z}\right) / \mathfrak{U}$ for some non-principal ultrafilter $\mathfrak{U}$ by Proposition 2.13(i). If $G$ is the limit of stationary sequence of finite cyclic groups, then $G$ is a finite cyclic group isomorphic to $\mathbb{Z} / k \mathbb{Z}$ for some $k \geq 1$. Then, we set $\mathfrak{U}$ to be the Dirac mass at $k$.

$(\Pi / \mathfrak{U})_{c} \Longrightarrow\left(T h_{\forall}\right)_{c}$ : as $G$ embeds into $\left(\prod_{n \geq 1} \mathbb{Z} / n \mathbb{Z}\right) / \mathfrak{U}$ for some ultrafilter $\mathfrak{U}$, we have $T h_{\forall}(G) \supset T h_{\forall}\left(\left(\prod_{n \geq 1} \mathbb{Z} / n \mathbb{Z}\right) / \mathfrak{U}\right)$. The result follows from Lŏs's theorem.

$\left(T h_{\forall}\right)_{c} \Longrightarrow(\lim )_{c}$ : as $T h_{\forall}(G) \supset \bigcap_{n \geq 1} T h_{\forall}(\mathbb{Z} / n \mathbb{Z})$, we have by Proposition 2.9 (ii) that $G$ is a limit in $\mathscr{M}$ of subgroups of cyclic groups. Hence $G$ is a limit of cyclic groups.

We now consider the left triangle.

$(\lim )_{c} \Longrightarrow(\text { iso })_{c}$ : as $G$ is a limit of cyclic groups, $G$ is abelian (recall that the property of being abelian is closed in $\mathscr{M}$ ). By the subgroup convergence lemma, the torsion subgroup 
$\operatorname{Tor}(G)$ of $G$ is itself a limit of cyclic groups. Because $\operatorname{Tor}(G)$ is finite, it is isolated in $\mathscr{M}$ (Remark 2.5). Any sequence converging to $\operatorname{Tor}(G)$ is then a stationary sequence. As a result, $\operatorname{Tor}(G)$ is cyclic.

$(\text { iso })_{c} \Longrightarrow(\text { res })_{c}$ : write $G=\mathbb{Z}^{r} \times \mathbb{Z} / k \mathbb{Z}$ with $r \geq 0$ and $k \geq 1$. Let $F$ be a finite subset of $G \backslash\{0\}$ and let $E \subset \mathbb{Z}$ be the set of all $i$-th coordinates of elements of $F$ for every $1 \leq i \leq r$. Choose distinct primes $p_{1}, \ldots, p_{r}$ such that each $p_{j}$ is coprime with all elements of $E \cup\{k\}$. The quotient map $q: G \rightarrow\left(\prod_{j=1}^{r} \mathbb{Z} / p_{j} \mathbb{Z}\right) \times \mathbb{Z} / k \mathbb{Z}$ defined in an obvious way has cyclic image by the Chinese Remainder Theorem. Clearly, no element of $F$ is mapped to the trivial element by $q$.

$(\text { res })_{c} \Longrightarrow(\lim )_{c}$ : follows from Remark2.6.

\section{Proof of Theorem 4.1.}

The first part of the proof is similar to the proof of Proposition 4.2. Actually, the following implications can be shown in the very same way:

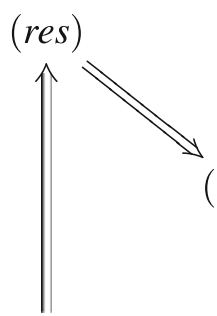

(iso)

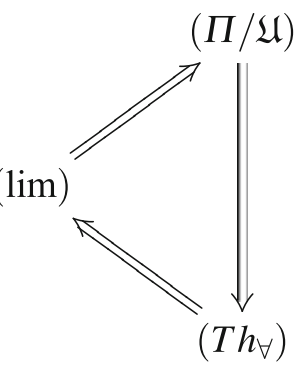

The only noticeable changes occur in the proof of $\left(T h_{\forall}\right) \Longrightarrow$ (lim): if $G$ is isomorphic to an abelian dihedral group then $G$ is obviously the limit of a stationary sequence of dihedral groups. Assume now that $G$ is not abelian. Since the property of being abelian is open in $\mathscr{M}$, the group $G$ is the limit of non-abelian subgroups of dihedral groups. Hence, $G$ is the limit of dihedral groups.

Then, we complete the proof by showing: $(\lim ) \Longrightarrow($ iso $)$. As $G$ is a limit of dihedral groups in $\mathscr{M}$, there is some marking $S$ of $G$ on $m$ generators and a sequence $\left(G_{n}, S_{n}\right)_{n}$ of marked dihedral groups such that $\left(G_{n}, S_{n}\right)_{n}$ converges to $(G, S)$ in $\mathscr{M}_{m}$. Let $Z(G)$ and $Z\left(G_{n}\right)$ denote the center of $G$ and $G_{n}$, respectively. We set $A:=\left\{g \mid g \in G, g^{2} \neq 1\right\} \cup Z(G)$ and $A_{n}:=\left\{g \mid g \in G_{n}, g^{2} \neq 1\right\} \cup Z\left(G_{n}\right)$. The group $A_{n}$ is a cyclic subgroup of index two in $G_{n}$. We denote by $p: \mathbb{F}_{m} \rightarrow G$ and $p_{n}: \mathbb{F}_{m} \rightarrow G_{n}$ the epimorphisms which map $\left(e_{i}\right)_{1 \leq i \leq m}$ to $S$ and $S_{n}$. We deduce from the convergence hypothesis that $p_{n}^{-1}\left(A_{n}\right)$ converges to $p^{-1}(A)$ in $\{0,1\}^{\mathbb{F}_{m}}$. As a result, $p^{-1}(A)$ is normal in $\mathbb{F}_{m}$ and hence $A$ is normal in $G$. We also deduce that $G_{n} / A_{n}$ tends to $G / A$ in $\mathscr{M}_{m}$. Since the set of marked groups of order 2 is a finite subset of $\mathscr{M}_{m}$, we have $[G: A]=2$.

We now consider $x \in \mathbb{F}_{m} \backslash p^{-1}(A)$ and $a \in p^{-1}(A)$. We have $x \in \mathbb{F}_{m} \backslash p_{n}^{-1}\left(A_{n}\right)$ and $a \in p_{n}^{-1}\left(A_{n}\right)$ for all $n$ large enough. Consequently $x a x^{-1}=a^{-1}$ holds in $G_{n}$ for all $n$ large enough. Thus $x_{a x}^{-1}=a^{-1}$ holds in $G$. Hence $G$ is isomorphic to the semi-direct product $A \rtimes \mathbb{Z} / 2 \mathbb{Z}$ where the non-trivial element of $\mathbb{Z} / 2 \mathbb{Z}$ acts by $a \mapsto a^{-1}$ on $A$.

It only remains to show that $A$ is a limit of cyclic groups. We fix a marking $T=\left(a_{1}, \ldots, a_{l}\right)$ of $A$ and elements $x_{j}$ such that $p\left(x_{j}\right)=a_{j}$. In the space $\mathscr{M}_{l}$, the marked group $(A, T)$ is the limit of $\left\langle p_{n}\left(x_{1}\right), \ldots, p_{n}\left(x_{l}\right)\right\rangle$ marked by $\left(p_{n}\left(a_{1}\right), \ldots, p_{n}\left(a_{l}\right)\right)$. As $x_{j} \in p^{-1}(A)$, we have $x_{j} \in p_{n}^{-1}\left(A_{n}\right)$ for all $n$ large enough. As a result, the subgroup $\left\langle p_{n}\left(x_{1}\right), \ldots, p_{n}\left(x_{l}\right)\right\rangle \leq A_{n}$ is cyclic and $A$ is a limit of cyclic groups. 


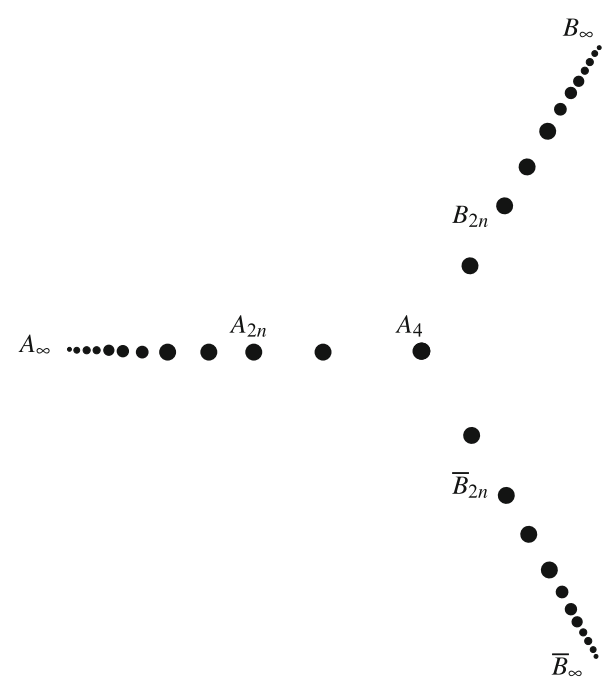

Fig. 1 Dihedral marked groups on 2 generators

\section{The space of limits of dihedral groups on $m$ generators}

Let $G=A \rtimes \mathbb{Z} / 2 \mathbb{Z}$ be a 2 -generated non-abelian limit of dihedral groups. As the rank of $A \rtimes \mathbb{Z} / 2 \mathbb{Z}$ is easily seen to be $r(A)+1$, the group $A$ is cyclic and hence $G$ is a dihedral.

Corollary 5.1 The space of dihedral marked groups on 2 generators is a closed and open subspace of $\mathscr{M}_{2}$.

\section{Proof}

It only remains to show that this subspace is open. As a quotient of a dihedral group is a dihedral group, the result follows from Lemma 2.4 .

We have a complete topological description of the space of dihedral groups on two generators. For each $n \neq 2$, there are exactly three distinct marked groups on two generators which are isomorphic to $\mathbb{D}_{2 n}$ :

$$
\begin{aligned}
A_{2 n} & :=\left\langle a, b \mid a^{2}=b^{2}=(a b)^{n}=1\right\rangle, \\
B_{2 n} & :=\left\langle a, b \mid a^{2}=b^{n}=1, a^{-1} b a=b^{-1}\right\rangle, \\
\bar{B}_{2 n} & :=\left\langle a, b \mid b^{2}=a^{n}=1, b^{-1} a b=a^{-1}\right\rangle .
\end{aligned}
$$

There is a unique marked group of $\mathscr{M}$ which is isomorphic to $\mathbb{D}_{4}: A_{4}=B_{4}=\bar{B}_{4}$. The only accumulation points in the space of dihedral groups on two generators are the three distinct marked infinite dihedral groups $A_{\infty}, B_{\infty}$ and $\bar{B}_{\infty}$ (see Fig. 1 below). This last fact is proved in Proposition 5.4. The remaining statements can be readily adapted from this proposition.

We can carry out such an analysis in the space of marked groups on $m$ generators, for any $m \geq 2$, by using Cantor-Bendixson invariants defined in Sect. 3 . We denote by $\omega$ the smallest infinite ordinal, i.e., the set $\mathbb{N}$ of positive integers endowed with its natural order.

Theorem 5.2 The topological closure $\mathscr{D}_{m}$ of dihedral marked groups in $\mathscr{M}_{m}$ is homeomorphic to $\omega^{m-1}\left(2^{m}-1\right)+1$ endowed with the order topology. 
By the Mazurkiewicz-Sierpinski Theorem (Sect.3), it suffices to show that the $(m-1)$-th derived set $\mathscr{D}_{m}^{(m-1)}$ of $\mathscr{D}_{m}$ contains $2^{m}-1$ points. This is carried out in the two following propositions:

Proposition 5.3 Let $G=A \rtimes \mathbb{Z} / 2 \mathbb{Z}$ be a limit of dihedral groups on $m$ generators. Then the Cantor-Bendixson rank of $G$ in $\mathscr{D}_{m}$ is the free rank of $A$.

Hence, the only remaining marked groups in $\mathscr{D}_{m}^{(m-1)}$ are marked groups abstractly isomorphic to $\mathbb{Z}^{m-1} \rtimes \mathbb{Z} / 2 \mathbb{Z}$. We now count them:

Proposition 5.4 Let $G=\mathbb{Z}^{m-1} \rtimes \mathbb{Z} / 2 \mathbb{Z}$ with $m \geq 2$. In $\mathscr{M}_{m}$, there are exactly $2^{m}-1$ marked groups which are abstractly isomorphic to $G$.

We show Propositions 5.3 and 5.4. Theorem 5.2 follows immediately.

Let $\mathscr{A} \subset \mathscr{M}$ be the set of abelian marked groups, let $\mathscr{D} \subset \mathscr{M}$ be the set of dihedral marked groups Let $\mathscr{D} \subset \mathscr{M}$ be the set of generalised dihedral marked groups $\operatorname{Dih}(A):=A \rtimes \mathbb{Z} / 2 \mathbb{Z}$ where $A$ is a finitely generated abelian group on which $\mathbb{Z} / 2 \mathbb{Z}$ acts by multiplication by -1 . Let $\mathscr{C}$ be the topological closure of cyclic marked groups in $\mathscr{M}$. We define $\operatorname{Dih}(A, S)$ with $S=\left(a_{1}, a_{2}, \ldots\right)$ as the marked group $\left(\operatorname{Dih}(A), S^{\prime}\right)$ with $S^{\prime}=\left(a, a_{1}, a_{2}, \ldots\right)$ where $a$ denotes the generator of $\mathbb{Z} / 2 \mathbb{Z}$.

The strategy of the proof of Proposition 5.3 is to exhibit a neighborhood of $(G, S)$ which is the image of an open injection from $\mathscr{C}$ into $\mathscr{D}$. As the Cantor-Bendixson rank of $(G, S)$ in $\mathscr{M}$ does not depend on $S$, we can fix a preferred marking $S$. We choose a marking whose first generator has order 2 .

Lemma 5.5 The map Dih $: \mathscr{A} \longrightarrow \tilde{D}$ is a continuous and open embedding. Moreover, if $(G, S) \in \mathscr{D}$ and $S$ is a marking of $G$ whose first generator has order 2 , then $(G, S) \in$ $\operatorname{Dih}(\mathcal{C}) \subset \mathscr{D}$.

\section{Proof of Lemma 5.5}

We fix words $v_{1}, \ldots, v_{k}, w_{1}, \ldots, w_{l}$ in $\mathbb{F}_{m+1}$ for which the exponent sum of $e_{1}$ is zero. We define the system

$$
(\Sigma):\left\{\begin{array}{l}
v_{1}=1, \ldots, v_{k}=1 \\
w_{1} \neq 1, \ldots, w_{l} \neq 1
\end{array}\right.
$$

Let

$$
D:=\left\langle e_{1}, \ldots, e_{m+1} \mid e_{1}^{2}=1, e_{1} e_{i} e_{1}^{-1}=e_{i}^{-1},\left[e_{i}, e_{j}\right]=1, i=2, \ldots, m+1\right\rangle .
$$

We reduce the words $v_{i}, w_{j}$ in $D$ for $i=1, \ldots, k$ and $j=1, \ldots, l$ to get words without symbols $e_{1}^{ \pm 1}$. We then shift the indices on the left $\left(e_{i}\right.$ becomes $\left.e_{i-1}\right)$ to obtain words $v_{i}^{\prime}, w_{j}^{\prime}$ in $\mathbb{F}_{m}$. We define $\left(\Sigma^{\prime}\right)$ by replacing $v_{i}$ by $v_{i}^{\prime}$ and $w_{j}$ by $w_{j}^{\prime}$ in $(\Sigma)$. We consider the elementary open sets $O_{\Sigma^{\prime}} \subset \mathscr{A}$ and $O_{\Sigma} \subset \mathscr{D}$. Let $(A, S)$ be in $\mathscr{A}$. It is trivial to check that $\operatorname{Dih}(A, S) \in O_{\Sigma} \Longleftrightarrow(A, S) \in O_{\Sigma^{\prime}}$. Hence $\operatorname{Dih}$ is a continuous and open map.

The injectivity of $\operatorname{Dih}$ is obvious. The inclusion $\operatorname{Dih}(\mathscr{C}) \subset \mathscr{D}$ follows from Theorem 4.1.

Lemma 5.6 Let $A$ be a finitely generated abelian group (respectively, a limit of cyclic groups). Then the Cantor-Bendixson rank of $A$ in $\mathscr{M}$ (respectively in $\mathscr{C}$ ) is the free rank of $A$. 


\section{Proof of Lemma 5.6}

First, we show that the Cantor-Bendixson of $A$ in $\mathscr{M}$ is not less than the free rank of $A$. If $A$ is torsion, it is trivial. Hence we can choose any $z \in A$ of infinite order and apply the induction hypothesis to $A /\left\langle z^{n}\right\rangle$ for any $n>0$. The result follows. If $A$ is in $\mathscr{C}$, we consider quotients of the form $A /\left\langle z^{n}\right\rangle$ where $n$ is coprime with the number of torsion elements of $A$.

It remains to show that the Cantor-Bendixson rank is not greater than the free rank. This follows from Lemma 2.4 which allows a straightforward induction on the free rank.

\section{Proof of Proposition 5.3}

Let $G=A \rtimes \mathbb{Z} / 2 \mathbb{Z}$ be a limit of dihedral groups on $m$ generators. As $(G, S)$ is in the image of $D i h$ for the choosen marking $S$, its Cantor-Bendixson rank in $\mathscr{D}_{m}$ is the Cantor-Bendixson rank of $A$ in $\mathscr{C}$ by Lemma 5.5. This is the free rank of $A$ by Lemma 5.6.

\section{Proof of Proposition 5.4}

For any $S=\left(g_{1}, \ldots, g_{m}\right)$ in $V(G, m)$, we define $I(S) \subset\{1, \ldots, m\}$ as the set of indices $i$ such that $g_{i}^{2}=1$. It is routine to check the following two facts:

- Let $S, S^{\prime} \in V(G, m)$. We have: $I(S)=I\left(S^{\prime}\right)$ if and only if $(G, S)$ is equivalent to $\left(G, S^{\prime}\right)$.

- For any non-empty subset $P$ of $\{1, \ldots, m\}$, there is $S \in V(G, m)$ such that $P=I(S)$.

The result follows.

\section{Remarks on the space $\mathscr{M}_{\infty}$ of countable groups}

In this section, we describe a natural topology on the space of marked countable groups. We then extend (partially) the characterisations of limits of cyclic groups given in Proposition 4.2 and the characterisations of limits of dihedral groups given in Theorem A to countable groups. All the claims in this section are elementary and proofs are left to the reader.

We have stated our main results in the setting of finitely generated groups because the topology of $\mathscr{M}$ seems to reflect more deeply group-theoretic properties than the space of countable groups does. Nevertheless, we give a definition of this space, as it is a more general object.

The pair $(G, S)$ is a marked countable group if $G$ is a countable group and $S=\left(g_{1}, g_{2}, \ldots\right)$ is an infinite sequence of generators of $G$. Two marked groups $(G, S)$ and $\left(G^{\prime}, S^{\prime}\right)$ (with $S=$ $\left(g_{1}, g_{2}, \ldots\right)$ and $\left.S^{\prime}=\left(g_{1}^{\prime}, g_{2}^{\prime}, \ldots\right)\right)$ are equivalent if there is an isomorphism $\phi: G \longrightarrow G^{\prime}$ such that $\phi\left(g_{i}\right)=g_{i}^{\prime}$ for $i=1,2, \ldots$ Let $\mathscr{M}_{\infty}$ be the set of equivalence classes of countable marked groups. By the universal property of $\mathbb{F}_{\infty}$, the set $\mathscr{M}_{\infty}$ naturally identifies with the set $\mathbb{N}\left(\mathbb{F}_{\infty}\right)$ of all normal subgroups of $\mathbb{F}_{\infty}$. We endow $\mathbb{N}\left(\mathbb{F}_{\infty}\right)$ with the topology induced by the product space $\{0,1\}^{\mathbb{F}}$ and hence define a topology on $\mathscr{M}_{\infty}$. We call this space the space of countable groups. Lemmas 2.3 and 2.7, Propositions 2.9 and 2.13 have obvious analogs in $\mathscr{M}_{\infty}$. The space $\mathscr{M}_{\infty}$ is easily shown to be metrisable, compact and totally disconnected. One pleasant and elementary fact about $\mathscr{M}_{\infty}$ is that the subset of countable marked groups $H$ such that $T h_{\forall}(H) \supset T h_{\forall}(G)$ for a given countable group $G$ coincides with the topological closure of all countable groups abstractly isomorphic to $G$.

While the topological space $\mathscr{M}$ seems difficult to describe, the space $\mathscr{M}_{\infty}$ can be clearly identified up to homeomorphism:

Proposition 6.1 The space $\mathscr{M}_{\infty}$ is a compactification of $\mathscr{M}$ homeomorphic to a Cantor set. 
The fact that $\mathscr{M}_{\infty}$ is perfect can be deduced from Lemmas 6.2 and 6.3 below.

The first lemma shows that some direct limits can be interpreted as limits in $\mathscr{M}_{\infty}$ :

Lemma 6.2 Let $G$ be the direct limit of a system

$$
G_{1} \underset{f_{1}}{\rightarrow} G_{2} \underset{f_{2}}{\rightarrow} \cdots \underset{f_{n-1}}{\rightarrow} G_{n} \underset{f_{n}}{\rightarrow} G_{n+1} \underset{f_{n+1}}{\rightarrow} \cdots
$$

where all the groups $G_{n}$ are finitely generated. Then there exist infinite markings $S$ and $S_{n}$ of $G$ and $G_{n}$ such that $(G, S)$ is the limit of $\left(G_{n}, S_{n}\right)_{n}$ in $\mathscr{M}_{\infty}$.

In particular, any non-finitely generated group is a limit of some sequence of its finitely generated subgroups in $\mathscr{M}_{\infty}$. Hence non-finitely generated marked groups are not isolated in $\mathscr{M}_{\infty}$. Neither are finitely generated marked groups because of the next lemma.

Lemma 6.3 Let $G$ be a countable group and let $H$ be a finitely generated subgroup of $G$. For any infinite marking $S$ of $H$, there are infinite markings $S_{n}$ of $G$ such that $(H, S)$ is the limit of $\left(G, S_{n}\right)_{n}$ in $\mathscr{M}_{\infty}$.

Since any finitely generated group embeds into a non-finitely generated countable group, finitely generated marked groups are indeed limit points in $\mathscr{M}_{\infty}$.

Proposition 4.2 has the following generalisation in $\mathscr{M}_{\infty}$ :

Proposition 6.4 Let $G$ be a countable group. The following conditions are equivalent:

$(\lim )_{\infty} G$ is a limit of cyclic groups in $\mathscr{M}_{\infty}$;

$(\text { iso })_{\infty} G$ is isomorphic to an abelian group whose torsion subgroup embeds isomorphically into $\mathbb{Q} / \mathbb{Z}$;

$\left(T h_{\forall}\right)_{c} T h_{\forall}(G) \supset \bigcap_{n \geq 1} T h_{\forall}(\mathbb{Z} / n \mathbb{Z})$;

$(\Pi / \mathfrak{U})_{c} \quad G$ is isomorphic to a subgroup of $\left(\prod_{n \geq 1} \mathbb{Z} / n \mathbb{Z}\right) / \mathfrak{U}$ for some ultrafilter $\mathfrak{U}$ on $\mathbb{N}$.

Characterisation (iso $)_{\infty}$ is equivalent to say that the torsion subgroup of $G$ is locally cyclic. Characterisation $(\text { res })_{c}$ of Proposition 4.2 is not valid in $\mathscr{M}_{\infty}$ because limits are not necessarily finitely presented. For instance, observe that $\mathbb{Q}$ is a limit of cyclic groups in $\mathscr{M}_{\infty}$ without being fully residually cyclic. An analog of Theorem A also holds in $\mathscr{M}_{\infty}$. The proof is a simple adaptation of Proposition 4.2 and Theorem A which uses Proposition 6.2. Arguing with Lemma 6.3, we can easily show that the topological closures of the set of cyclic groups and dihedral groups in $\mathscr{M}_{\infty}$ are both homeomorphic to a Cantor set. The corresponding closures in $\mathscr{M}$, are readily shown to be homeomorphic to $\omega^{\omega}$ endowed with the order topology (it immediately follows from [15, Prop. 3.2]).

Acknowledgments I am particularly grateful to Yves Stalder, who considerably improved the proof of Theorem B. I thank Pierre de La Harpe, Goulnara Arzhantseva and Catriona Maclean for their valuable comments on previous versions of this article. I also thank Thierry Coulbois and Vincent Guirardel for stimulating discussions.

Open Access This article is distributed under the terms of the Creative Commons Attribution Noncommercial License which permits any noncommercial use, distribution, and reproduction in any medium, provided the original author(s) and source are credited.

\section{References}

1. Bell, J.L., Slomson, A.B.: Models and Ultraproducts: An Introduction. North Holland, Amsterdam (1969) 
2. Champetier, C., Guirardel, V.: Limit groups as limits of free groups. Isr. J. Math. 146, 1-75 (2005)

3. Chabauty, C.: Limite d'ensembles et géométrie des nombres. Bull. Soc. Math. France 78, 143-151 (1950)

4. Champetier, C.: L'espace des groupes de type fini. Topology 39(4), 657-680 (2000)

5. Cornulier, Y., Guyot, L., Pitsch, W.: On the isolated points in the space of groups. J. Algebra 307(1), 254277 (2007)

6. de la Harpe, P.: Topics in Geometric Group Theory. Chicago Lectures in Mathematics. University of Chicago Press, Chicago (2000)

7. Grigorchuk, R.: Degrees of growth of finitely generated groups and the theory of invariant means. Izv. Akad. Nauk SSSR Ser. Mat. 48(5), 939-985 (1984)

8. Grigorchuk, R.: Solved and unsolved problems around one group. In: Infinite Groups: Geometric, Combinatorial and Dynamical Aspects, volume 248 of Progr. Math., pp. 117-218. Birkhäuser, Basel (2005)

9. Gromov, M.: Groups of polynomial growth and expanding maps. Inst. Hautes Études Sci. Publ. Math. 53, 53-73 (1981)

10. Guirardel, V.: Limit groups and groups acting freely on $\mathbb{R}^{n}$-trees. Geom. Topol. 8, 1427-1470 electronic (2004)

11. Kharlampovich, O., Myasnikov, A.: Irreducible affine varieties over a free group I. Irreducibility of quadratic equations and Nullstellensatz. J. Algebra 200(2), 472-516 (1998)

12. Kharlampovich, O., Myasnikov, A.: Irreducible affine varieties over a free group. II. Systems in triangular quasi-quadratic form and description of residually free groups. J. Algebra 200(2), 517-570 (1998)

13. Mazurkiewicz, S., Sierpinski, W.: Contribution à la topologie des ensembles dénombrables. Fund. Math. 1, 17-27 (1920)

14. Neumann, H.: Varieties of Groups. Springer, New York (1967)

15. Pierce, R.S.: Existence and uniqueness theorems for extensions of zero-dimensional compact metric spaces. Trans. Am. Math. Soc. 148, 1-21 (1970)

16. Sela, Z.: Diophantine geometry over groups I. Makanin-Razborov diagrams. Publ. Math. Inst. Hautes Études Sci. 93, 31-105 (2001)

17. Shalom, Y.: Rigidity of commensurators and irreducible lattices. Invent. Math. 141(1), 1-54 (2000)

18. Stepin, A.: Approximation of groups and group actions, the Cayley topology. In: Ergodic theory of $\mathbf{Z}^{d}$ actions (Warwick, 1993-1994), volume 228 of London Math. Soc. Lecture Note Ser., pp. 475-484. Cambridge University Press, Cambridge (1996) 